\title{
Cell therapy for type 1 diabetes
}

\author{
Cristian Loretelli, Emma Assi, Andy Joe Seelam, Moufida Ben Nasr and Paolo Fiorina
}

\section{QUERY SHEET}

This page lists questions we have about your paper. The numbers displayed at left are hyperlinked to the location of the query in your paper.

The title and author names are listed on this sheet as they will be published, both on your paper and on the Table of Contents. Please review and ensure the information is correct and advise us if any changes need to be made. In addition, please review your paper as a whole for typographical and essential corrections.

Your PDF proof has been enabled so that you can comment on the proof directly using Adobe Acrobat. For further information on marking corrections using Acrobat, please visit http://journalauthors.tandf.co.uk/production/acrobat.asp; https://authorservices.taylorandfrancis.com/how-to-correct-proofs-with-adobe/

The CrossRef database (www.crossref.org/) has been used to validate the references. Changes resulting from mismatches are tracked in red font.

\section{AUTHOR QUERIES}

Q1 References were not cited sequentially. Hence, references have been renumbered from Ref.70. Please check.

Q2 Please check the correctness of the term 'immunologlobulin'.

Q3 Reference. 70 was a duplicate of Reference. 81 and hence has been deleted and subsequent references and their respective citations have been renumbered accordingly. Please confirm if this is correctly done. 


\title{
Cell therapy for type 1 diabetes
}

\author{
Cristian Loretellia*, Emma Assia*, Andy Joe Seelama ${ }^{a}$ Moufida Ben Nasra,b and Paolo Fiorina ${ }^{a, b, c}$
}

anternational Center for T1D, Pediatric Clinical Research Center "Romeo Ed Enrica Invernizzi", Department of Biomedical and Clinical Science

5 L. Sacco, Università Degli Studi Di Milano, Milan, Italy; 'bephrology Division, Boston Children's Hospital, Harvard Medical School, Boston, MA, USA; 'Division of Endocrinology, ASST Fatebenefratelli-Sacco, Milan, Italy

\section{ABSTRACT}

Introduction: Type 1 diabetes (T1D) is a lifelong condition resulting from autoimmune destruction of insulin-producing $\beta$-cells. Islet or whole-pancreas transplantation is limited by the shortage of donors and need for chronic immune suppression. Novel strategies are needed to prevent $\beta$-cell loss and to rescue production of endogenous insulin.

Areas covered: This review covers the latest advances in cell-based therapies for the treatment and prevention of T1D. Topics include adoptive transfer of cells with increased immunoregulatory potential for $\beta$-cell protection, and $\beta$-cell replacement strategies such as generation of insulin-producing $\beta$-like cells from unlimited sources.

Expert opinion: Cell therapy provides an opportunity to prevent or reverse T1D. Adoptive transfer of autologous cells having enhanced immunomodulatory properties can suppress autoimmunity and preserve $\beta$-cells. Such therapies have been made possible by a combination of genome-editing techniques and transplantation of tolerogenic cells. In-vitro modified autologous hematopoietic stem cells and tolerogenic dendritic cells may protect endogenous and newly generated $\beta$-cells from a patient's autoimmune response without hampering immune surveillance for infectious agents and malignant cellular transformations. However, methods to generate cells that meet quality and safety standards for clinical applications require further refinement.
ARTICLE HISTORY

Received 30 October 2019

Accepted 25 March 2020

\section{KEYWORDS}

Cell therapy; T1D; Treg therapy; HSC therapy; tolerogenic dendritic cells; immunotherapy; $\beta$-cell replacement; ESC-derived $\beta$-cells; iPSC-derived $\beta$-cells

\section{Introduction}

Current treatment regimen for patients with type 1 and 2 diabetes mellitus (T1D and T2D, respectively) largely relies on administration of exogenous insulin and glucose-sensing technology. Although beneficial for improving glycometabolic control, insulin replacement remains limited by its inherent inability to fully replicate the biological functions of endogenous insulin; this places patients at risk for hypoglycemic episodes [1]. Patients with T1D have a considerably reduced life expectancy because T1D is exacerbated by long-term macro and microvascular complications [2]. Restoration of full endocrine pancreatic function is met in a relatively small number of patients via transplantation of cadaveric whole pancreas or islets. However, the clinical success of such procedures is limited by poor post-transplant engraftment of vascular cells, blood-mediated inflammatory response, hypoxia ${ }_{\text {and }}$ hypoxia-reoxygenation injury, and alloimmune response against the graft $[2,3]$. Furthermore, islets transplanted into T1D patients are also exposed to persistent autoimmune attacks directed against islet autoantigens. Therefore, much effort has been focused on developing immunosuppressive strategies aimed at providing immune protection to grafted islets. Although safer and more effective anti-inflammatory agents have been developed, the need for chronic immune suppression places transplant recipients at a considerable risk for severe infections, cancer, and $\beta$-cell damage [4-6]. This highlights the need for immunosuppressant-free strategies in islet transplantation. One of these strategies involves encapsulation of islet cells within biocompatible semi-impermeable membranes or capsular devices, which create a physical and functional barrier between the graft and patient's immune cells, while simultaneously allowing the diffusion of oxygen and nutrients, and promoting favorable insulin-secretion kinetics (reviewed in Navarro-Tableros V. et al., 2018 [7]). In human patients, however, this approach did not provide durable immunoprotection to the graft mainly because of membrane damage, instability of biomaterials used in construction of the device, and poor oxygen supply to the graft [8]. Therefore, islet transplantation is in need of novel therapeutic strategies not requiring chemical immunosuppression. The low long-term survival rate of islet grafts, side effects associated with chronic immunosuppression, and shortage of donors providing pancreas and islets restricts the availability of these therapies to few selected patients with severe hypoglycemia or instable T1D. Moreover, most patients with T1D require transplants from more than one donor in order to achieve a clinically relevant improvement of their condition [9].

Islet immunoprotection, freedom from iatrogenic immunosuppression, and unlimited availability of functional $\beta$-cells/pancreatic islets are the goals of achieving independence from exogenous insulin administration in T1D. One of the most promising areas in diabetes research involves the development 
Article Highlights

- A definitive cure for T1D is centered around protecting $\beta$-cells from autoimmunity and on regenerating/replacing $\beta$-cells.

- All approaches designed to stop $\beta$-cell disruption have failed, while transplantation of pancreas or pancreatic islets obtained from deceased donors cannot meet the growing demand.

- Regulatory T cells, hematopoietic stem cells, and tolerogenic dendritic cells can be turned into islet-targeting immunoprotective tools in vitro, and adoptively transferred into patients with T1D to preserve residual $\beta$-cells and rescue $\beta$-cell function.

- ESC- or iPSC-derived cells are an unlimited source of functionally active $\beta$-like cells that can be engineered to escape recipient's alloimmune and autoimmune reactions.

- Generating cells endowed with enhanced immunosuppressive properties and capable of immunoevasion holds great potential for $\beta$-cell immunopreservation and replacement in T1D cell therapies.

This box summarizes key points contained in the article.

of different in-vitro or ex-vivo modified cells that can be used to

75 replace disrupted $\beta$-cells, or combined with $\beta$-cells and delivered via injection. Unlike immunotherapies, which have been studied extensively, cell-based therapies have only recently been brought to the forefront of diabetes research, and a considerable number of studies are still in progress. This is

80 reflected by the lower number of published scientific reports on these therapies. Here we review the most recent advances and future perspectives in the field of cell therapies for T1D, including the latest progresses in cell-based $\beta$-cell immunoprotection and $\beta$-cell replacement strategies.

\section{2. $\beta$-cell preservation by regulatory T cell (Treg)} therapy

Islet autoantigen-specific $\mathrm{CD}^{+}$and $\mathrm{CD}^{+}$effector $\mathrm{T}$ cells are major contributors to $\beta$-cell destruction in T1D. These autoreactive $T$ cells are normally deleted during thymic selection, and the

90 few escapees are held in check in the periphery by a multitude of peripheral tolerance mechanisms. Among these, Foxp3expressing $\mathrm{CD}^{+}$regulatory $\mathrm{T}$ cells (Tregs) play fundamental roles in controlling autoreactive cells and are, thus, central to T1D pathophysiology [10]. Numerous studies have examined

95 Tregs in T1D patients and in non-obese diabetic (NOD) preclinical mouse models, but results are not unanimous. While there is no defect in Treg frequency in T1D, impaired Treg phenotypes, and functionality have been shown by several groups [11,12], but refuted by others [13]. However, studies in humans and mice indicate that increased activity of effector T cells and defects in Tregs may be the factors driving T1D. New interventions, aimed at correcting this imbalance and increasing the number of Tregs in circulation, show promising preliminary results [10]. Two phase I clinical trials, examining polyclonal Treg adoptive immunotherapy in recent-onset patients with T1D, have been published thus far [14-16]. In these studies, autologous $\mathrm{CD} 4{ }^{+} \mathrm{CD} 127^{\mathrm{lo} /-} \mathrm{CD} 25^{+}$polyclonal Tregs were isolated from patients' peripheral blood, expanded ex-vivo with anti-CD3, antiCD28, and interleukin (IL) 2, and reinfused into the donors at achieved in both studies, and reinfused polyclonal Tregs were

found to be phenotypically stable and persistent in circulation 1 year after treatment $[14,16]$. However, although an initial improvement in glycemic control was observed, $C$ peptide levels progressively diminished over time. The limitations of these studies included insufficient numbers of treated participants and a need for a longer follow up. A phase II study on Treg therapy in T1D (ClinicalTrials.gov identifier: NCT02691247) and a phase I study on Tregs combined with low-dose IL-2 administration (ClinicalTrials.gov identifier: NCT02772679) are currently ongoing.

Despite these encouraging results, Treg infusion can only temporarily delay the progression of T1D. The main challenge lies in overcoming several key factors that limit the efficacy of Treg immunotherapy. First, the stage of disease progression at the time of treatment is pivotal. Because $\beta$-cell reservoir decreases during the course of T1D, earlier interventions preserve a greater number of $\beta$-cells from autoimmune attack. Moreover, disease stage also affects Treg immunoregulatory activity. Analysis of this cell population suggests that progression of the disease is accompanied by decreases in Treg number and function. Therefore, it is advisable to use autologous Tregs as early as possible in order to obtain an optimal preparation of Tregs for clinical applications [17]. Strategies aimed at enhancing the efficacy of adoptive Treg therapies include increasing Treg numbers, using antigen-specific Tregs, and enhancing Treg stability, survival, and function. Future clinical trials should be focused on the development of Tregs with $\beta$-cell antigen specificity to maximize: (i) islet homing and therapeutic index, and (ii) retention of Tregs over time to obtain a durable response [18]. Expanding autoantigen-specific Treg populations would specifically block autoimmune destruction of $\beta$-cells without affecting other components of the immune system. Moreover, islet antigen-specific clones may preferentially home to inflamed islets and expand locally to exert site-specific effects. The potential protective effect of autoantigen-specific Tregs on $\beta$-cells was confirmed in pre-clinical studies, in which Tregs, challenged with islet-specific peptides/major histocompatibility complex (MHC) assemblies, were more effective in reversing autoimmune diabetes than were polyclonal Tregs in a mouse model of T1D $[19,20]$. Nevertheless, generating large populations of Tregs with islet-antigen specificity in humans remains debated, calling into question the translational power of this approach. In a recent report, Hull et al. generated large numbers of human islet antigen-specific Tregs via lentiviral T-cell receptor (TCR) gene transfer. However, these Tregs were less responsive to cognate antigens than to viral-specific TCRs [21]. The clinical practicability of this approach is limited by restrictions of the major histocompatibility complex $(\mathrm{MHC})$, requiring that each patient would need the transduction of a tailored TCR. In an elegant work, Tenspolde et al. generated functionally active insulin-specific Tregs using chimeric antigen receptor-T cell (CAR-T) technology to obtain CAR-T regulatory cells (CAR-Tregs) [22]. These cells were generated using lentiviral transduction of $\mathrm{CD}^{+}$murine effector T cells with a chimeric antigen receptor vector system; this system contained insulin-specific $\mathrm{T}$ cell activation domains, costimulatory molecules, and a Foxp3 Treg marker having immunoregulatory functions. The stable expression of Foxp3 in these cells transformed $\mathrm{T}$ effectors into converted $\mathrm{T}$ regulatory cells (cTregs). Insulin-specific cTregs are phenotypically and 
functionally similar to natural Tregs, and show long lifespans in diabetic mice. However, insulin-specific cTregs cannot prevent the onset of diabetes [22]. Generating effective CAR-Tregs is still in its infancy, and further studies are needed to improve this promising approach. This technique, however, may potentially be used to overcome the challenges involved in $\mathrm{MHC}$ matching and may offer alternatives to in-vitro Treg expansion.

Antigen-specific Tregs can also be expanded directly in vivo, avoiding in-vitro cell manipulations. Combined treat-

180 ment using $\mathrm{MHC}$ /peptide molecules and IL-2/anti-IL-2 monoclonal antibody complexes has recently been shown to induce a rapid expansion of antigen-specific Foxp $3^{+}$Tregs, accompanied by the proliferation of an antigen-specific Foxp3- populations producing anti-inflammatory IL-10. This approach effectively prevents the onset of T1D in mice [23].

Targeting Tregs for T1D prevention and treatment is a promising, but new, area of diabetes research. Detailed understanding of antigen-specific Tregs in humans will help optimize protocols for boosting the numbers and/or functions of these cells before using them in actual therapies for patients with T1D.

\section{Immunoregulatory hematopoietic stem cells (HSCs)}

The use of immunoregulatory hematopoietic stem cells (HSCs) involves promising but challenging strategies for the treatment of T1D. Indeed, sufficient metabolic control, indicated by independence from exogenous insulin, decreased $\mathrm{HbA} 1 \mathrm{c}$ levels, and increased C-peptide levels, has been successfully achieved using HSC treatment in a significant proportion of treated patients with T1D $[24,25]$. Multipotent mesenchymal stem cells (MSCs) of different origins, alone or combined with encapsulating devices, have also been successfully used to prevent or revert diabetes in murine models of T1D [26-28]. In clinical trials, however, MSC-based therapies show limited success in overall metabolic control compared with that of placebo-treated groups [29]. Furthermore, the potential tumorigenicity of MSCs in the long term has not been completely ruled out, posing concerns over their clinical use. In a preclinical setting, Sertoli cells have also been evaluated for their immunoregulatory properties, and have been successfully used to reverse hyperglycemia in overtly diabetic NOD mice [30]. Thus far,

210 the most encouraging effects were obtained with autologous nonmyeloablative hematopoietic stem cell transplantation (AHSCT). Half of individuals receiving this therapy achieved insulin independence, which was sustained long term in $40 \%$ of the treated arm [24]. However, this treatment is associated with serious adverse events, which highlight the need for considerable improvements to this therapeutic approach. A multicenter crosssectional analysis study, performed in patients with T1D treated with AHSCT and followed up for 8 years, showed that this patient cohort was free from major diabetic complications (macrovascular and microvascular), while over $21 \%$ of placebo-treated group experienced microvascular complications [31]. Although not without limitations, AHSCT remains one of the most promising therapies to successfully revert T1D. Refining this therapeutic strategy will improve its clinical outcomes. Thus far, absence of long-term patient follow-up and lack of a control group or placebo-treated arm, as well as vast variability in inclusion criteria, are considered major limitations of this approach [32]. As shown by the first published clinical trials, the use of AHSCT in autoimmune diseases is aimed to halt the function of autoreactive cells and establish immune self-tolerance [33]. To determine the mechanisms involved in this therapy, studies have used next-generation sequencing on TCR B chains in order to assess and sequence whole-patient TCR repertoire before and after AHSCT [34]. The results show that $\mathrm{CD} 4^{+}$and $\mathrm{CD} 8^{+} \mathrm{T}$ cell clones following $\mathrm{AHSCT}$ were substantially different from those at baseline in this patient population. Interestingly, patient-dominant $\mathrm{CD}^{+}{ }^{+} \mathrm{T}$ cell clones, reportedly present before treatment, were undetectable after immune reconstitution. This indicates that these cells were successfully eliminated by the therapy. $\mathrm{CD}^{+} \mathrm{T}$ cell clones were not completely removed, indicating that the patients' newly reconstituted $\mathrm{CD}^{+} \mathrm{T}$ cell clones were generated by clonal expansion of the remnant clones [34]. Further analysis showed that the nonresponder group displayed lesser diversity within their TCR clones early in the reconstitution phase. This suggests that patients receiving AHSCT can be monitored according to their TCR profile; however, this notion requires further confirmation in larger clinical trials. In clinical trials conducted on other autoimmune diseases, patients with systemic lupus erythematosus treated with AHSCT and respective controls were immunophenotypically characterized at 24 months post-treatment. Comparing the status of these patients at 24 months post-treatment with their baseline parameters indicated regeneration of recent thymic emigrants, which were highly detectable within the responder group. This suggests that regeneration of the Treg compartment occurred within the thymus [35]. This is also unclear. I suggest: "Patients with relapsing multiple sclerosis treated with AHSCT also demonstrated attenuation of pro-inflammatory response." This was accompanied by a dramatic depletion of mucosal-associated invariant T (MAIT) cells, which are active in inflammatory activity, and increased numbers of Tregs and CD56 ${ }^{\text {high }}$ natural killer (NK) cells, which show immunoregulatory properties [36]. AHSCT exerts numerous beneficial effects in responders, particularly in longterm responders, who experience a prolonged period of insulin independence and are protected from the risk of developing diabetic complications [31,37]. Overall, the beneficial effects and metabolic control achieved with AHSCT therapy may be due to the degree of islet autoreactivity that patients exhibit prior to receiving this therapy. In fact, patients with low autoreactivity to cytotoxic $T$ lymphocytes $(C T L)$ remain insulin independent in the long term and show higher C-peptide levels after therapy with AHSCT compared with those having high CTL autoreactivity. Recurrence of diabetes in patients with high CTL autoreactivity is mainly due to incomplete depletion of autoreactive T cells, which can undergo clonal expansion [37]. Thus, precision-targeting of the conditioning therapy may be critical for achieving improved results and effective reversal of the disease. The immunoregulatory potential of HSCs has recently been examined in NOD mice, in which experimental autoimmune diabetes was reverted. Because patients with T1D and NOD mice possess defective HSCs, overcoming this defect using genetic engineering successfully converted hyperglycemia to normoglycemia in NOD mice and inhibited the autoimmune response. This approach opens new venues for interventions based on lentiviral-delivered gene therapy. Indeed, such an approach may reduce or eliminate the need for the conditioning regimen used in AHSCT and provides the rationale for conducting ad hoc clinical trials to test this hypothesis 
[38]. Patients' disease status at baseline is another important criterion affecting ASHCT therapy because the extent of preexisting $\beta$-cell mass can impact the outcomes of AHSCT. Several

290 post-hoc studies analyzing clinical trials performed with AHSCT confirmed the highly positive correlation between baseline and post-AHSCT C-peptide levels. Because C-peptide levels reflect the functional status of $\beta$-cell mass, fasting $C$-peptide level is an effective predictor of long-term remission in patients with T1D

295 after AHSCT therapy. Patients without any history of diabetic ketoacidosis (DKA) that are treated within 6 months of diagnosis generally show long-term reversal of T1D. Future trials should evaluate young and pediatric patients who have a high capacity for $\beta$-cell regeneration and may, therefore, be good candidates for

300 AHSCT therapy $[24,38]$.

\section{Tolerogenic dendritic cells (tolDCs)}

Dendritic cells (DCs) are antigen-presenting, cells (APC) that perform immune surveillance and provide costimulatory signals during $\mathrm{T}$ cell priming [39]. Maturational and functional

305 abnormalities in DCs have been demonstrated in patients with T1D and in NOD mice [40-43]. Indeed, mature DCs can secrete IL-12, thereby stimulating a $\mathrm{T}$ helper 1 (Th1) response. Immature DCs expressing low levels of costimulatory molecules induce antigen-specific hyporesponsiveness by promot-

310 ing T cell anergy/apoptosis and expansion of Tregs, inducible Tregs, and regulatory B cells (Bregs) [43-46]. Numerous recent studies have examined tolerogenic dendritic cells (tolDCs), which show considerable promise in therapies against autoimmune diseases $[47,48]$. Clare-Slazler et al. [49] reported that

315 DCs isolated from pancreatic lymph nodes of normoglycemic NOD mice prevented the onset of diabetes when injected into 4-week-old NOD mice. This prompted further investigation of establishing DC-based therapies for T1D. Despite the laborintensive process required to generate tolDCs in vitro, this cell 320 type offers many advantages compared to other ex-vivo generated cells [47]. TolDCs act as a central hub for regulation of the immune response because they can target Tregs, lack the clonality of $T$ cells, and can migrate into the main inflammatory sites [50,51]. Protocols using granulocyte macrophage-

325 colony stimulating factor (GM-CSF)/IL-4 or GM-CSF/IL-10 have been developed to streamline the process of tolDC expansion and generation in vitro [52,53]. The aforementioned properties of tolDCs render these cells potentially useful in cell-based therapy, and they are currently being evaluated in several clinical trials $[50,54]$. The safety of using tolDCs to suppress autoimmunity was assessed in a randomized phase 1 clinical trial conducted in patients with T1D. This trial compared immunosuppressive toIDCs generated ex-vivo with a placebo control group. The results of that study indicated that treatment with tolDCs was well tolerated in patients with T1D, showed a good safety profile, and may portend several beneficial outcomes [55]. Furthermore, patients that received tolDCs showed increased levels of Breg enriched $\mathrm{B}_{2} 2 \mathrm{O}^{+}$ CD11 $\mathrm{c}^{-}$cell populations compared with those of placebo340 treated controls. This phenomenon has already been described in a preclinical setting, in which tolDCs successfully reverted T1D in NOD mice, thereby increasing the frequency of IL-10-producing B cells [56]. ToIDCs can be generated using different methods including using oligonucleotides conjugated to specific costimulatory molecules (CD40, CD80, CD86); co-culture with inhibitors of nuclear factor-kB (NF-kB), dexamethasone, and vitamin D3 or A; or pre-loading tolDCs with disease-specific auto-antigens to enhance their interactions with autoreactive T cells. Despite the diversity of methods used for tolDCs generation, these cells mostly contribute to increased frequency of circulating Tregs and decreased levels of Th1/Th17-associated cytokines (such as interleukins 12,15 , and 23) [57,58]. Nevertheless, the status of metabolic control in patients with T1D plays a critical role in their response to toIDC therapy and may affect the functionality of these cells [43]. Dexamethasone/vitamin D2-modulated tolDCs, obtained from patients with poor metabolic control and from patients with well-controlled glycemia, both show immunoregulatory and anti-inflammatory abilities independent of their origin. Interestingly, those originating from patients with well-controlled glycemia express higher levels of the inhibitory molecules immunologlobulin-like transcript 3 and PD-L1. Compared with toIDCs derived from patients with poor metabolic control, GAD65-loaded tolDCs isolated from patients with well-controlled glycemia show higher immunosuppressive responses, which significantly decreases Th1- and Th17-mediated responses, and induces a sustained GAD65specific $\mathrm{T}$ cell hyporesponsiveness. Altogether, these results emphasize the importance of metabolic control in patients with T1D. The state of metabolic control should be taken into consideration, especially when defining the main inclusion criteria in future clinical trials for T1D [43].

\section{5. $\beta$-cell replacement therapy using embryonic stem cells (ESCs)}

Human embryonic stem cells (ESCs) are considered the gold standard for pluripotent stem cell sources used in cell therapies. These cells are collected from the inner cell mass of human blastocysts and can be indefinitely maintained or in-vitro differentiated into cells of virtually any tissue type, including $\beta$-cell-like cells. ESC-derived $\beta$-cells were first successfully generated by D'Amour et al. [59] via the in-vitro recapitulation of pancreatic islets and $\beta$-cells physiological development by stepwise application of specific factors. During normal embryonic development, future $\beta$-cells transition from the definitive endoderm through stages encompassing the primitive gut tube, posterior foregut, multipotent pancreatic progenitors, endocrine precursors, and immature endocrine cells. These cells then finally differentiate into functional mature $\beta$-cells, each characterized by distinct features and cell-identity markers. In their differentiation protocol, D'Amour et al. exposed a monolayer culture of ESCs to a series of key growth factors and signaling molecules, used at specific doses and in a defined sequence, to guide the cells through a sequential five-stage process mimicking the natural $\beta$ cell development. Cells obtained at the end of this process were polyhormonal, showed faulty insulin secretion in response to glucose sensing, and were more similar to immature pancreatic endocrine cells rather than to mature $\beta$-cells. However, once transplanted into immunocompromised mice, these immature cells spontaneously differentiated into functional singlehormone - secreting endocrine cells, and were able to rescue 
normoglycemia in streptozotocin-induced diabetic mice after $2-3$ months $[60,61]$. Since then, several groups have substantially improved this in-vitro differentiation protocol by optimizing the type of growth factors used, and timing of growth-factor and 405 cytokine administration [62]. Overall, all ESC differentiation protocols rely on: activation or inhibition of growth factors and molecular pathways (such as WNT, TGF- $\beta$, Sonic Hedgehog, FGFs, BMPs, Notch, thyroid hormones, and PKC); controlling cell proliferation, differentiation, and identity; and using suspension

410 cultures with controlled stirring to promote cell-cell interactions and formation of islet-like cell aggregates [63-65]. These improved protocols generate increased yields of insulinpositive cells with improved glucose responsiveness and decreased numbers of polyhormonal cells. Notably, cells gener415 ated using these improved methods successfully restored glycemic control in immunocompromised diabetic mice at only 2-6 weeks from transplantation [63,64]. Additional recently proposed optimizations to the ESC differentiation protocol include YAP inhibition [66], release from TGF- $\beta$ inhibition [67], and delayed inhibition of the WNT pathway [68].

ESC differentiation toward the mature $\beta$-cell phenotype is tracked at each stage by monitoring the expression of markers identifying each intermediate progenitor, such as endocrine precursor marker NEUROG3; a-cell markers GCG, ETV1, and 425 ARX; and $\beta$-cell markers insulin, NKX6.1, and PDX1 [68]. Singlecell RNA sequencing analysis has shown that cells in the first three stages make up a uniform population; however, from the third stage and beyond, the culture becomes heterogeneous, and multiple arising subpopulations include $\alpha-, \beta$-cells, and scant numbers of enteroendocrine-like cells $[68,69]$.

Although considerable advances have been made in generating $\beta$-cells from ESCs, critical hurdles still preclude the clinical use of ESCs. Compared with that of islet $\beta$-cells, ESCderived $\beta$-cells display a variable, but persistently lower, expression of crucial $\beta$-cell markers including that of GPM6A, MAFA, HDAC9, IAPP, and HADH; this is a symptom of incomplete maturation, which accounts for the observed suboptimal glucose responsiveness and $\beta$-cell function [67]. Moreover, the use of embryo-derived stem cells is complicated by ethical 440 concerns, while other limitations of ESC usage include potential development of teratomas and presence of heterogeneous populations among generated cells including polyhormonal and other types of undesirable non-islet cells [70]. The most pressing issue remains ESC-derived $\beta$-cell graft rejection, which is caused by both alloimmune and persistent autoimmune responses, rendering recipients dependent on vigorous immunosuppression [8]. Encapsulation, which is also used for islet transplantation, can help prevent immune attacks on ESCderived $\beta$-cell grafts and may also physically contain potentially developing teratomas. A phase $1 / / l$ clinical trial was conducted in 2014 by ViaCyte to test the safety and efficacy of ESC-derived pancreatic progenitor cells encased in immunoisolating macrocapsules $\left(\mathrm{VC}-01^{\mathrm{tm}}\right)$ and transplanted into patients with T1D (ClinicalTrials.gov identifier: NCT02239354).

455 Despite encouraging results with respect to immunoprotection, however, prolonged cell survival failed to meet expectations [71], re-emphasizing the need for novel and more effective strategies for $\beta$-cell immunopreservation. Microencapsulation devices may enable the delivery and immunoprotection of islets or $\beta$-cells with no need for immunosuppression. These devices are smaller in size than macrocapsules, and their greater surface/volume ratio facilitates the exchange and diffusion of nutrients, oxygen, and hormones with the surrounding tissues. Preclinical $[27,72]$ and clinical studies [73,74], mainly employing alginate-based microcapsules, have shown encouraging results, but materials, preparation $_{a}$ and purification methods, employed in the manufacturing of these devices, still need to be standardized and optimized [75].

\section{Induced pluripotent stem cells (iPSCs) as sources for $\beta$-cell replacement therapy}

Induced pluripotent stem cells (iPSCs), discovered by Takahashi and Yamanaka in 2006 and 2007, have reinvigorated the field of cell/regenerative therapy. iPSC technology has enabled the reprogramming of murine and human fibroblasts, as well as other adult somatic cells, into pluripotent cells by delivering genes encoding three or four transcription factors such as Oct3/4, Sox2, Klf4, c-Myc, Lin28, and/or Nanog $[76,77]$. The traits of newly-generated iPSCs are similar to those of ESCs in terms of morphology, functionality, and expression of pluripotency-associated factors. Similar to ESCs, iPSCs can be induced to differentiate into numerous cell types, including insulin-producing cells [78]. Using ESCs in cell therapy is mainly limited by ethical concerns over the destruction of embryos to generate ESCs, as well as by possible triggering of the alloimmune response associated with the usage of ectopic ESC-derived cells. iPSCs overcome these limitations because they can be reprogrammed directly from one individual's adult cells, thereby allowing the generation of patientderived pluripotent cell lines that do not trigger the recipient's alloimmune responses [79].

The differentiation protocols used to generate ESCs are typically used with several modifications to generate iPSCs. However, the process of differentiating iPSCs into $\beta$-cells remains a major challenge, and includes the usage of various cytokines and signaling molecules to activate or inhibit pathways playing key roles in the function, maintenance, and differentiation of $\beta$-cells [80]. Several studies have optimized the maturation process of pancreatic progenitor cells by first culturing iPSCs in a 2D matrigel platform and then in suspension culture. The resultant pancreatic progenitor cells were then transplanted into immunodeficient mice, in which they further differentiate and mature into glucose-responsive insulin-secreting cells [70]. Melton et al. optimized in-vivo differentiation of iPSCs into insulin-producing pancreatic $\beta$-like cells by first generating high levels of NKX6.1- and PDX1-positive pancreatic progenitor clusters. Next, the cells were cultured for 4-5 weeks and administered 11 factors influencing various pathways to generate $\beta$-cells capable of responding to a glucose challenge in vitro and in vivo $[64,81]$. Nonetheless, maturation of progenitor endocrine cells and transplantation of in-vitro - formed $\beta$-cells remain highly controversial due to numerous factors such as formation of polyhormonal cells lacking $\beta$-cell properties and persistence of undifferentiated iPSCs having a tendency to form teratomas. Conversely, 
transplantation techniques used for ESCs, and use of implantation or encapsulating devices made of biocompatible materials such as alginate derivatives, may promote the survival, differentiation, and maturation of pancreatic cells, and limit teratoma development [82].

Generating iPSCs with high efficiency and good safety profile remains a key objective in the long-term therapeutic application of these cells. However, despite improved differentiation protocols, efficient iPSC generation still requires the

525 usage of retro/lentiviral methods of transduction, which overexpresses oncogenic genes such as C-MYC and KLF4, while random genomic integration associated with virus-based delivery methods carries a risk of increased mutational load leading to mutagenesis and tumorigenesis $[83,84]$. Current

530 efforts to resolve this issue include the use of selfinactivating vectors (SIN), designed to inactivate oncogenic genes after iPSCs are generated, and resulting in generation of committed cells in which pluripotency is lost [85]. Other techniques include producing iPSCs with decreased genotoxic 535 potential for reprogramming target cells, and usage of nonintegrating adenoviral or Sendai virus vectors, purified proteins, transposons, modified RNAs, and miRNAs [86]. The usage of such non-integrating methods is still infrequent due to their low efficiency and high cost. Additionally, the auto-

540 logous nature of any iPSCs that fail to fully differentiate during the differentiation process, along with possible accumulation of DNA alterations arising from in-vitro cell manipulation, still pose a risk of potentially tumorigenic cells escaping immunosurveillance. Eliminating potentially tumorigenic cells during

545 iPSC derivation involves screening undifferentiated iPSCs using surface antigen markers for pluripotency [87] and inhibition of cellular overgrowth by treatment with $\gamma$-secretase [88]. Recent studies by Itakura et al. have shown that induction of caspase- 9 expression in conditional engineered iPSCs can be used as a genetic fail-safe mechanism by inducing apoptosis in tumor-prone, undifferentiated cells [89]. In recent years, genome-editing tools, such as CRISPR/CAS9, have been developed and used extensively in iPSC-based applications. Such usage includes correcting disease-driving mutations by deletion, substitution, and insertions in conditions such as Duchenne muscular dystrophy, frontotemporal dementia, and common syndromes associated with inherited intellectual disability. Although not yet used in $\beta$-cell generation, this technology can pave the way for genomic corrections in mutated iPSCs, producing healthy $\beta$-cells that can be transplanted into patients [90]. Despite these advances, techniques used to obtain and transplant in-vitro generated $\beta$-cells still need to be optimized with respect to transplantation, maturation, survivability, genetic stability, and cost of generation.

565 Incomplete de-differentiation of iPSCs is another important factor that needs to be addressed. In this process, the identity of original cells is not completely reset, and they may carry an 'epigenetic memory' of the original cell type from which they differentiated. This phenomenon may impair the reprogramming efficiency, functionality, and teratoma-forming potential of these iPSCs [91].

Various preclinical studies can successfully generate insulinproducing pancreatic $\beta$-cells from human iPSCs. However, these $\beta$-cells have not been transplanted into human patients, and no clinical trials have examined transplantation of iPSCderived pancreatic $\beta$-cells into human participants. This is mainly because the protocols used for iPSC reprogramming, differentiation, maturation, and transplantation cannot yet produce clinically acceptable insulin-producing $\beta$-cells $[92,93]$.

The use of islet organoids is a promising therapeutic strategy for the treatment of T1D. Organoids are a group of primary cells or iPSCs grown in vitro and allowed to form 3D structures having similar functionality and organization to those of pancreatic islets in vivo. In recent years, various groups have generated islet-like clusters/aggregates, as well as islet-like organoids, derived from iPSCs [70]. Various culturing systems, using a combination of biomaterials such as matrigel, laminin, fibronectin, and decellularized scaffolds, have been used to assist in the formation of functional $\beta$-cell niches incorporating endocrine cells. Transplantation of such vascularized islet-like organoids into type 1 diabetic mice has considerably improved graft survival and normalized blood glucose levels [70]. This promising approach, however, is still critically limited by poor vasculogenesis and nerve formation around the engraftment, which reduces the functionality of the graft [70].

Using iPSCs in anti-T1D therapy appears promising, as rapid advances in stem-cell technology, immune modulation, encapsulation techniques, and protocols for generation of $\beta$ cells from iPSCs have already overcome numerous challenges. To fully realize their potential clinical use, further improvements need to be made to increase the safety and efficacy of iPSCs for use in a clinical setting.

\section{Conclusion}

Cell-based therapies can provide alternatives to lifelong insulin administration in patients with T1D (Figure 1). If used in the early stages of the disease, when a significant portion of $\beta$-cell mass is still present, adoptive transfer or in-vivo expansion of islet antigen-specific Tregs, AHSCT therapy, and administration of tolerogenic DCs represent promising cell-based therapies that may effectively preserve $\beta$-cell function. Once $\beta$-cells are lost, however, physiological glycemic control can only be achieved by replacing the degenerated cells. ESCs or iPSCs, as an unlimited source for in-vitro generation of $\beta$-cells, may obviate the shortage of pancreas/islet donors. However, strategies to prevent graft rejection and teratoma formation are still needed before iPSC-based therapies can be translated into clinical use.

\section{Expert opinion}

Cell therapy is a breakthrough technology expected to revolutionize therapeutic concepts in multiple fields including immunotherapy, regenerative medicine, and cancer. Nextgeneration genome-editing techniques, such as CRISP/Cas9based methods, have enabled the production of engineered cells, endowed with desirable biological properties, that can be adoptively transferred into patients.

In the context of T1D, best-case scenarios involve combining cell-based therapies with genome editing to generate cells 


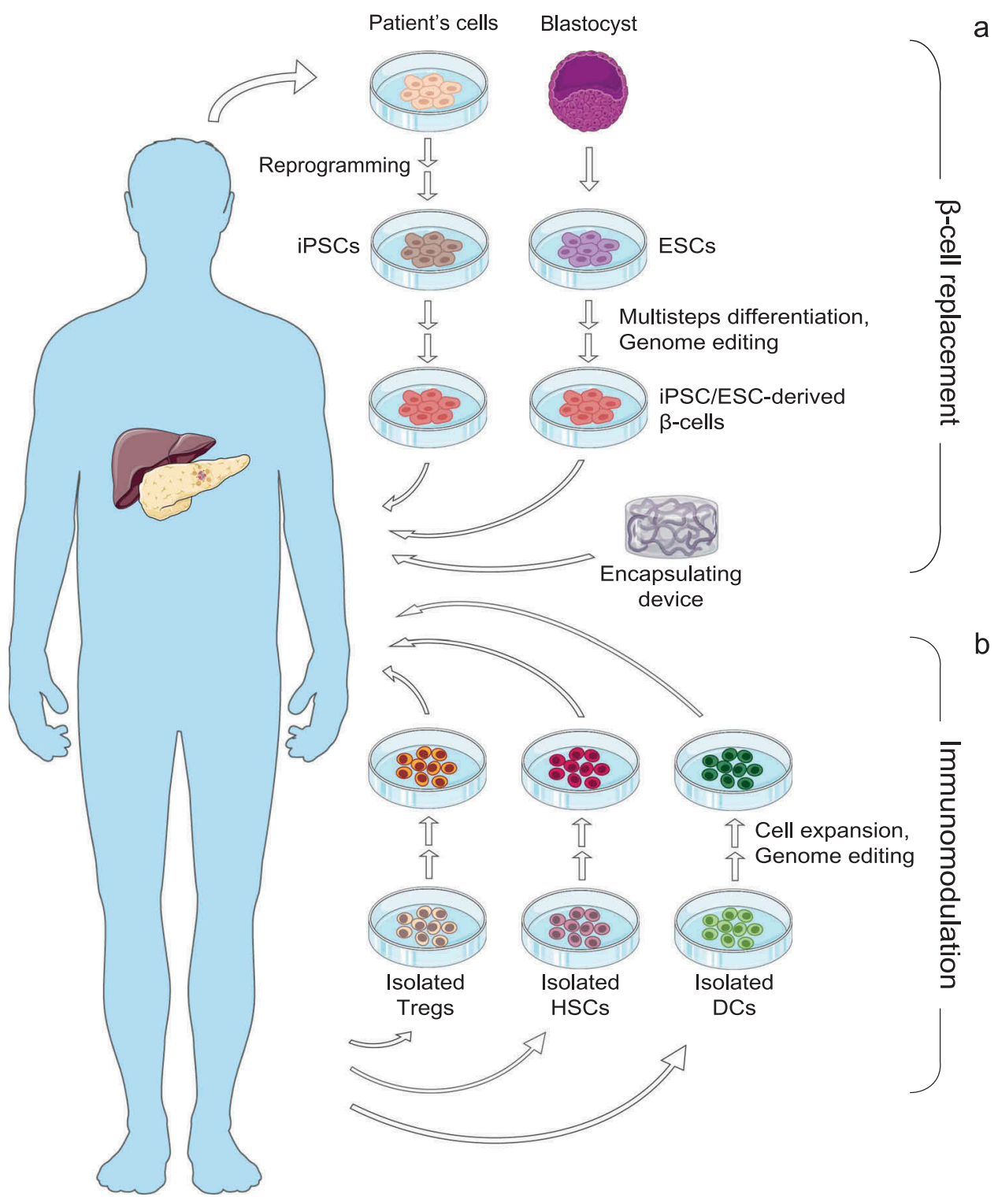

Figure 1. Schematic of cell therapy interventions in T1D. T1D is characterized by autoimmune destruction of $\beta$-cells. (a) Once $\beta$-cell mass has decreased below a critical level, $\beta$-like cells, generated in vitro from ESCs or patient-derived iPSCs, are transplanted into T1D patients to restore $\beta$-cell function. Genetic modifications and encapsulating technologies may help preserve these transplanted cells from the recipient's immune response and may prevent teratoma formation. (b) In young patients, or in those in early phases of T1D, cell-based therapeutic interventions are aimed at protecting the residual viable $\beta$-cells from autoimmune attack. Approaches used for this purpose include expansion of patients' autoantigen-specific Treg clones, and in-vitro modification of patients' own HSCs or generation of tolerogenic DCs used to induce self-tolerance in the recipient. Modified immunomodulatory cells are then autologously re-injected into the cell donor to provide immunoprotection to $\beta$-cells.

able to blunt autoimmunity and/or restore $\beta$-cell function. Residual remaining $\beta$-cells can be protected from autoimmune attacks by techniques involving collection, in-vitro manipulation, and expansion of the patient's own Tregs to generate stable autoantigen-specific Tregs. Islet antigen-specific Tregs target $\beta$-cells directly and have a greater chance of islet homthese Tregs over time to obtain a durable response. Yet, the need to obtain $\mathrm{MCH}$-matched Tregs from each patient renders this approach challenging in terms of cost and time requirements, while methods used to generate sufficient numbers of antigen-specific Tregs need to be further optimized prior to clinical use. In this regard, cTregs are advantageous for producing universal cells that can be administered into patients with T1D regardless of their MCH haplotype. However, preclinical

studies show that cTreg-based therapies cannot restore normoglycemia, which limits the use of these therapies in the clinic.

Although still in their infancy, techniques for ex-vivo DC modification to generate tolDCs may serve as additional novel tools in therapies against autoimmune diseases including T1D. The safety of in-vitro enhanced tolDCs has already been shown, and in-vitro cell programming protocols are still being optimized. Culture conditions for toIDC generation from patient-derived DCs are currently being improved using different combinations of growth factors, interleukins, hormones, and vitamins. The use of oligonucleotides targeting immunocostimulatory molecules has been shown to enhance interactions between DCs and autoreactive $T$ cells and to significantly dampen Th1 and Th17 responses. Islet peptide-loaded tolDCs can effectively decrease 
the responsiveness of peptide-specific T cells. In this regard, advances in genome-editing techniques will help to further improve immunomodulatory properties in tolDCs by generating tolDCs that specifically target islet autoantigen-specific $T$ cells.

Among cell-based therapies, AHSCT may realistically enable T1D prevention or reversal in the near future by reestablishing long-term self-immune tolerance while preserving alloimmune response. This will furnish insulin independence without losing immunoprotection against exogenous infectious agents in patients with T1D. A particularly promising strategy for patients in the early stages of T1D involves ex-vivo modification of a patient's HSCs to obtain cells that overexpress important immune checkpoint proteins, and then autologously transplanting these cells into the patient. Additional benefits of this approach include reducing or even eliminating the stressful conditioning regimen currently needed in AHSCT for abrogating autoreactive $\mathrm{CD}^{+}$and $\mathrm{CD} 8^{+} \mathrm{T}$ cell clones. This will protect the patients from exposure to life-threatening pathogenic infections with no concurrent risk for tumor formation.

When $\beta$-cells are lost completely, cell therapy can restore the natural autonomous glycometabolic control. ESCs or iPSCs can be differentiated into $\beta$-cells and transplanted into T1D patients with prolonged disease. ESCs are currently being tested in clinical trials for the treatment of several conditions including spinal cord, injury, Parkinson's disease, and diabetes; however, no clinical trials using iPSCs have been initiated thus far. The challenges that need to be overcome when developing ESC/PSC-based therapies for T1D include suboptimal quality and functionality of in-vitro differentiated $\beta$-cells, exposure of transplanted cells to alloimmune and autoimmune reactions, and risk of teratoma formation by ESC/iPSC-derived cells [94]. While protocols for stem-cell differentiation and scalability are rapidly evolving, standardized procedures for yielding highly functional $\beta$-cells are still lacking and are urgently needed. The question of whether generating a pure population of $\beta$-cells is truly the optimal choice for obtaining metabolically functional insulin-releasing cells remains unanswered. Indeed, a pool of different pancreatic-islet endocrine cells, structured into organoids mimicking native islets, may better reproduce the islet cell-cell interaction and yield superior performance overall.

Genome editing may pave the way for controlling the immunogenicity of transplanted cells. ESCs and iPSCs can be in-vitro modified by reshaping their HLA class I and II profiles, and by inducing the overexpression of immunoregulatory factors such as PD-L1 and CD-47 [95]. The resulting cells would be invisible to both T- and NK-cell responses in all patients, thereby providing an off-the-shelf universal cell product that would be protected against acute rejection and chronic inflammatory response, and would eliminate the need for immunosuppression.

710 The main concern in ESC/iPSC-derived $\beta$-cell transplantation involves the potential risk for teratoma formation. The main risk factors for malignant transformation of grafted cells include, incomplete cell differentiation; in-vitro cell reprogramming, differentiation, and expansion; leaky expression of reprogramming

715 genes; and pronounced immune-evasion capabilities. Encapsulating technology can potentially confine uncontrolled cell spreading and protect transplanted cells from the recipient's immune-, cellular-, antibody-, and complement-mediated attack. However, the first Viacyte immunoisolating device that underwent clinical testing for ESC-derived $\beta$-cell transplantation did not show the expected results in terms of graft survival, prompting the launch of a second trial by the same company (ClinicalTrials.gov identifier: NCT03163511). In this second trial, chemical immunosuppression was reintroduced, emphasizing the need for improved encapsulating strategies and alternative approaches. In an elegant work, Qadir et al. generated ESCderived cells equipped with a double fail-safe mechanism to both prevent tumorigenesis and enrich differentiated cells for insulin-producing cells [96]. Cells were engineered with two suicide gene cassettes; therefore, only cells with telomerase activity (a marker of undifferentiated state and tumorigenic proliferation) and those with no insulin expression were killed by exposure to two specific chemical compounds, leaving alive only differentiated insulin-secreting cells. This system dampened the tumorigenic potential of differentiated cells in immunodeficient mice, with no apparent impairment of $\beta$-cell functionality. Similar strategies, expected in the near future, will prevent both teratoma formation and graft immunogenicity. Persistence of autoimmunity against islet peptides, however, remains a major issue, even when de-novo insulin-producing cells are introduced into the patient to replace dead $\beta$-cells. Therefore, in the context of $\beta$ cells replacement, tolerogenic cells, such as autoantigen-specific Tregs, tolDCs, and autologous HSCs, can be co-transplanted with ESC/PSC-derived cells to establish an immunoprotective cellular milieu required for a successful engraftment and long-term graft survival. The combination of genetic modifications and encapsulation technology represents a time-consuming and costly, yet promising, approach to a safe and effective use of ESC- or iPSCderived $\beta$-cells in the clinic. Yet, a tighter control of genome editing and genetic modification tools, standardization of cell differentiation procedures and improvement of safety profiles of cell-based therapies are still warranted. The autologous transplantation of Tregs, DCs, and especially HSCs with enhanced immunomodulatory properties is a factual option in T1D management. This approach can be used to both prevent the autoimmune destruction of $\beta$-cells and to assist engraftment and survival of transplanted $\beta$-cells having exogenous origins.

\section{Funding}

P Fiorina is supported by the Italian Ministry of Health grant RF-201602362512 and by the Linea-2 2019 funding from Università degli Studi di Milano. We thank the Fondazione Romeo and Enrica Invernizzi for the extraordinary support.

\section{Declaration of interest}

The authors have no relevant affiliations or financial involvement with any organization or entity with a financial interest in or financial conflict with the subject matter or materials discussed in the manuscript. This includes employment, consultancies, honoraria, stock ownership or options, expert testimony, grants or patents received or pending, or royalties.

\section{Reviewer Disclosures}

Peer reviewers on this manuscript have no relevant financial relationships 770 or otherwise to disclose. 


\section{References}

Papers of special note have been highlighted as either of interest $(\cdot)$ or of considerable interest $(\cdot \cdot)$ to readers.

1. Nathan DM, Genuth S, Lachin J, et al. The effect of intensive treatment of diabetes on the development and progression of long-term complications in insulin-dependent diabetes mellitus. N Engl J Med. 1993 Sep 30;329(14):977-986. PubMed PMID: 8366922.

780 2. Pepper AR, Bruni A, Shapiro AMJ. Clinical islet transplantation: is the future finally now? Curr Opin Organ Transplant. 2018 Aug;23 (4):428-439. PubMed PMID: 29847441.

3. Bassi R, Fiorina P. Impact of islet transplantation on diabetes complications and quality of life. Curr Diab Rep. 2011 Oct;11 (5):355-363. PubMed PMID: 21748256.

4. Nir T, Melton DA, Dor Y. Recovery from diabetes in mice by beta cell regeneration. J Clin Invest. 2007 Sep;117(9):2553-2561. PubMed PMID: 17786244; PubMed Central PMCID: PMCPMC1957545.

790 5. Shapiro AM, Pokrywczynska M, Ricordi C. Clinical pancreatic islet transplantation. Nat Rev Endocrinol. 2017 May;13(5):268-277. PubMed PMID: 27834384.

6. Ben Nasr M, D'Addio F, Usuelli V, et al. The rise, fall, and resurgence of immunotherapy in type 1 diabetes. Pharmacol Res. 2015 Aug;98:31-38. PubMed PMID: 25107501.

7. Navarro-Tableros V, Gomez Y, Brizzi MF, et al. Generation of human stem cell-derived Pancreatic Organoids (POs) for regenerative medicine. New York, NY: Springer; 2019. p. 1-42.

8. Sneddon JB, Tang Q, Stock $P$, et al. Stem cell therapies for treating diabetes: progress and remaining challenges. Cell Stem Cell. 2018 Jun 1;22(6):810-823. PubMed PMID: 29859172; PubMed Central PMCID: PMCPMC6007036.

9. Liu X, Li X, Zhang N, et al. Engineering beta-cell islets or islet-like structures for type 1 diabetes treatment. Med Hypotheses. 2015 Jul;85(1):82-84. PubMed PMID: 25892491.

10. Spence A, Tang Q. Restoring regulatory T cells in Type 1 diabetes. Curr Diab Rep. 2016 Nov;16(11):110. PubMed PMID: 27664043.

11. Brusko TM, Wasserfall $\mathrm{CH}$, Clare-Salzler MJ, et al. Functional defects and the influence of age on the frequency of CD4+ CD25+ T-cells in type 1 diabetes. Diabetes. 2005 May;54(5):1407-1414. PubMed PMID: 15855327.

12. Kukreja A, Cost G, Marker J, et al. Multiple immuno-regulatory defects in type-1 diabetes. J Clin Invest. 2002 Jan;109(1):131-140. PubMed PMID: 11781358; PubMed Central PMCID: PMCPMC150819.

13. Putnam AL, Vendrame $F$, Dotta $F$, et al. $C D 4+C D 25$ high regulatory T cells in human autoimmune diabetes. J Autoimmun. 2005 Feb;24 (1):55-62. PubMed PMID: 15725577.

14. Bluestone JA, Buckner JH, Fitch $M$, et al. Type 1 diabetes immunotherapy using polyclonal regulatory T cells. Sci Transl Med. 2015 Nov 25;7(315):315ra189. PubMed PMID: 26606968; PubMed Central PMCID: PMCPMC4729454.

15. Bluestone JA, Tang Q. Treg cells-the next frontier of cell therapy. Science. 2018 Oct 12;362(6411):154-155. PubMed PMID: 30309932.

16. Marek-Trzonkowska N, Mysliwiec M, Dobyszuk A, et al. Therapy of type 1 diabetes with CD4(+)CD25(high)CD127-regulatory T cells prolongs survival of pancreatic islets - results of one year follow-up. Clin Immunol. 2014 Jul;153(1):23-30. PubMed PMID: 24704576 .

830 17. Marek-Trzonkowska N, Mysliwiec M, Iwaszkiewicz-Grzes D, et al. Factors affecting long-term efficacy of $T$ regulatory cell-based therapy in type 1 diabetes. J Transl Med. 2016 Dec 1;14(1):332. PubMed PMID: 27903296; PubMed Central PMCID: PMCPMC5131539.

18. Visperas A, Vignali DA. Are regulatory T cells defective in Type 1 diabetes and can we fix them? J Immunol. 2016 Nov 15;197 (10):3762-3770. PubMed PMID: 27815439; PubMed Central PMCID: PMCPMC5119643.

19. Masteller EL, Warner MR, Tang Q, et al. Expansion of functional endogenous antigen-specific CD4+CD25+ regulatory $T$ cells from nonobese diabetic mice. J Immunol. 2005 Sep 1;175(5):3053-3059. PubMed PMID: 16116193.

20. Tarbell KV, Petit L, Zuo X, et al. Dendritic cell-expanded, islet-specific CD4+ CD25+CD62L+ regulatory T cells restore normoglycemia in diabetic NOD mice. J Exp Med. 2007 Jan 22;204 (1):191-201. PubMed PMID: 17210729; PubMed Central PMCID: PMCPMC2118426.

21. Hull CM, Nickolay LE, Estorninho M, et al. Generation of human islet-specific regulatory T cells by TCR gene transfer. J Autoimmun. 2017 May;79:63-73. PubMed PMID: 28117148.

22. Tenspolde M, Zimmermann K, Weber LC, et al. Regulatory T cells engineered with a novel insulin-specific chimeric antigen receptor as a candidate immunotherapy for type 1 diabetes. J Autoimmun. 2019 Jun 5:102289. PubMed PMID: 31176558. DOI:10.1016/j. jaut.2019.05.017

23. Izquierdo $C$, Ortiz AZ, Presa $M$, et al. Treatment of T1D via optimized expansion of antigen-specific Tregs induced by IL-2/anti-IL-2 monoclonal antibody complexes and peptide/MHC tetramers. Sci Rep. 2018 May 25;8(1):8106. PubMed PMID: 29802270; PubMed Central PMCID: PMCPMC5970271.

-. This paper provides an efficient protocol for expanding antigen-specific Foxp $3^{+}$Tregs and antigen-specific Foxp $3^{-}$populations directly in-vivo, resulting in prevention of T1D onset in mice.

24. D'Addio F, Valderrama Vasquez A, Ben Nasr M, et al. Autologous nonmyeloablative hematopoietic stem cell transplantation in new-onset type 1 diabetes: a multicenter analysis. Diabetes. 2014 Sep;63(9):3041-3046. PubMed PMID: 24947362.

25. Couri $C E$, Oliveira $M C$, Stracieri $A B$, et al. $C$-peptide levels and insulin independence following autologous nonmyeloablative hematopoietic stem cell transplantation in newly diagnosed type 1 diabetes mellitus. JAMA. 2009 Apr 15;301(15):1573-1579. PubMed PMID: 19366777.

26. Ben Nasr M, Vergani A, Avruch J, et al. Co-transplantation of autologous MSCs delays islet allograft rejection and generates a local immunoprivileged site. Acta Diabetol. 2015;52:917-927.

27. Montanucci $P$, Pescara T, Alunno A, et al. Remission of hyperglycemia in spontaneously diabetic NOD mice upon transplant of microencapsulated human umbilical cord Wharton jelly-derived mesenchymal stem cells (hUCMS). Xenotransplantation. 2019 Mar;26(2):e12476. PubMed PMID: 30552781.

28. Ohmura Y, Tanemura M, Kawaguchi N, et al. Combined transplantation of pancreatic islets and adipose tissue-derived stem cells enhances the survival and insulin function of islet grafts in diabetic mice. Transplantation. 2010 Dec 27;90(12):1366-1373. PubMed PMID: 21076379

29. Carlsson PO, Schwarcz E, Korsgren O, et al. Preserved beta-cell function in type 1 diabetes by mesenchymal stromal cells. Diabetes. 2015 Feb;64(2):587-592. PubMed PMID: 25204974.

30. Fallarino F, Luca G, Calvitti M, et al. Therapy of experimental type 1 diabetes by isolated Sertoli cell xenografts alone. J Exp Med. 2009 Oct 26;206(11):2511-2526. PubMed PMID: 19822646; PubMed Central PMCID: PMCPMC2768846.

31. Penaforte-Saboia JG, Montenegro RM Jr., Couri CE, et al. Microvascular complications in Type 1 diabetes: a comparative analysis of patients treated with autologous nonmyeloablative hematopoietic stem-cell transplantation and conventional medical therapy. Front Endocrinol (Lausanne). 2017:8:331. PubMed PMID: 29218029; PubMed Central PMCID: PMCPMC5703738.

- This is an interesting clinical report highlighting the protective role of autologous HSC transplantation against the risk of developing diabetic complications.

32. van Megen KM, van ' $T$ Wout ET, Forman SJ, et al. A future for autologous hematopoietic stem cell transplantation in Type 1 diabetes. Front Immunol. 2018;9:690. PubMed PMID: 29696017; PubMed Central PMCID: PMCPMC5904498.

33. Ben Nasr M, Bassi R, Usuelli V, et al. The use of hematopoietic stem cells in autoimmune diseases. Regen Med. 2016 Jun;11(4):395-405. PubMed PMID: 27165670. 
34. Muraro PA, Robins $H$, Malhotra $S$, et al. T cell repertoire following autologous stem cell transplantation for multiple sclerosis. J Clin Invest. 2014 Mar;124(3):1168-1172. PubMed PMID: 24531550; PubMed Central PMCID: PMCPMC3934160.

35. Alexander T, Thiel A, Rosen $\mathrm{O}$, et al. Depletion of autoreactive immunologic memory followed by autologous hematopoietic stem cell transplantation in patients with refractory SLE induces long-term remission through de novo generation of a juvenile and tolerant immune system. Blood. 2009 Jan 1;113(1):214-223. PubMed PMID: 18824594.

36. Darlington PJ, Touil T, Doucet JS, et al. Diminished Th17 (not Th1) responses underlie multiple sclerosis disease abrogation after hematopoietic stem cell transplantation. Ann Neurol. 2013 Mar;73 (3):341-354. PubMed PMID: 23463494.

37. Malmegrim KC, de Azevedo JT, Arruda LC, et al. Immunological balance is associated with clinical outcome after autologous hematopoietic stem cell transplantation in Type 1 diabetes. Front Immunol. 2017;8:167. PubMed PMID: 28275376; PubMed Central PMCID: PMCPMC5319960.

38. Ben Nasr M, Tezza S, D'Addio F, et al. PD-L1 genetic overexpression or pharmacological restoration in hematopoietic stem and progenitor cells reverses autoimmune diabetes. Sci Transl Med. 2017 Nov 15;9(416). PubMed PMID: 29141886; PubMed Central PMCID: PMCPMC6171337. DOI:10.1126/scitranslmed.aam7543.

39. Mukherjee G, Dilorenzo TP. The immunotherapeutic potential of dendritic cells in type 1 diabetes. Clin Exp Immunol. 2010 Aug;161 (2):197-207. PubMed PMID: 20491789; PubMed Central PMCID: PMCPMC2909402.

40. Serreze DV, Gaskins HR, Leiter EH. Defects in the differentiation and function of antigen presenting cells in NOD/Lt mice. J Immunol. 1993 Mar 15;150(6):2534-2543. PubMed PMID: 8450229.

41. Vasquez AC, Feili-Hariri M, Tan RJ, et al. Qualitative and quantitative abnormalities in splenic dendritic cell populations in NOD mice. Clin Exp Immunol. 2004 Feb;135(2):209-218. PubMed PMID: 14738447; PubMed Central PMCID: PMCPMC1808940.

42. Morel PA. Dendritic cell subsets in type 1 diabetes: friend or foe? Front Immunol. 2013 Dec 6;4:415. PubMed PMID: 24367363; PubMed Central PMCID: PMCPMC3853773.

43. Danova K, Grohova A, Strnadova P, et al. Tolerogenic dendritic cells from poorly compensated Type 1 diabetes patients have decreased ability to induce stable antigen-specific $T$ cell hyporesponsiveness and generation of suppressive regulatory T cells. J Immunol. 2017 Jan 15;198(2):729-740. PubMed PMID: 27927966.

44. Maldonado RA, von Andrian UH. How tolerogenic dendritic cells induce regulatory $T$ cells. Adv Immunol. 2010;108:111-165. PubMed PMID: 21056730; PubMed Central PMCID: PMCPMC3050492.

45. Morel PA, Turner MS. Dendritic cells and the maintenance of self-tolerance. Immunol Res. 2011 Aug;50(2-3):124-129. PubMed PMID: 21717078.

46. Kleffel $S$, Vergani $A$, Tezza $S$, et al. Interleukin-10+ regulatory $B$ cells arise within antigen-experienced $C D 40+B$ cells to maintain tolerance to islet autoantigens. Diabetes. 2015 Jan;64(1):158-171. PubMed PMID: 25187361; PubMed Central PMCID: PMCPMC4274804.

47. Stojanovic I, Dimitrijevic M, Vives-Pi M, et al. Cell-based Tolerogenic Therapy, Experience from Animal Models of Multiple Sclerosis, Type 1 Diabetes and Rheumatoid Arthritis. Curr Pharm Des. 2017;23 (18):2623-2643. PubMed PMID: 28201972.

48. Funda DP, Palova-Jelinkova L, Golias J, et al. Optimal Tolerogenic Dendritic Cells in Type 1 Diabetes (T1D) Therapy: what Can We Learn From Non-obese Diabetic (NOD) Mouse Models? Front Immunol. 2019;10:967. PubMed PMID: 31139178; PubMed Central PMCID: PMCPMC6527741.

49. Clare-Salzler MJ, Brooks J, Chai A, et al. Prevention of diabetes in nonobese diabetic mice by dendritic cell transfer. J Clin Invest. 1992 Sep;90(3):741-748. PubMed PMID: 1522229; PubMed Central PMCID: PMCPMC329925.

50. Creusot RJ, Giannoukakis N, Trucco M, et al. It's time to bring dendritic cell therapy to type 1 diabetes. Diabetes. 2014 Jan;63
(1):20-30. PubMed PMID: 24357690; PubMed Central PMCID: PMCPMC3968436.

51. Worbs T, Hammerschmidt SI, Forster R. Dendritic cell migration in health and disease. Nat Rev Immunol. 2017 Jan;17(1):30-48. PubMed PMID: 27890914.

52. Feili-Hariri M, Falkner DH, Gambotto A, et al. Dendritic cells transduced to express interleukin-4 prevent diabetes in nonobese diabetic mice with advanced insulitis. Hum Gene Ther. 2003 Jan 1;14 (1):13-23. PubMed PMID: 12573055.

53. Tai N, Yasuda $\mathrm{H}$, Xiang $Y$, et al. IL-10-conditioned dendritic cells prevent autoimmune diabetes in NOD and humanized HLA-DQ8/ RIP-B7.1 mice. Clin Immunol. 2011 Jun;139(3):336-349. PubMed PMID: 21458378

54. Ten Brinke A, Hilkens CM, Cools N, et al. Clinical use of tolerogenic dendritic cells-harmonization approach in european collaborative effort. Mediators Inflamm. 2015;2015:471719. PubMed PMID: 26819498; PubMed Central PMCID: PMCPMC4706930.

55. Giannoukakis N, Phillips B, Finegold D, et al. Phase I (safety) study of autologous tolerogenic dendritic cells in type 1 diabetic patients. Diabetes Care. 2011 Sep;34(9):2026-2032. PubMed PMID: 21680720; PubMed Central PMCID: PMCPMC3161299.

56. Di Caro V, Phillips B, Engman C, et al. Involvement of suppressive B-lymphocytes in the mechanism of tolerogenic dendritic cell reversal of type 1 diabetes in NOD mice. PLoS One. 2014;9(1): e83575. PubMed PMID: 24465383; PubMed Central PMCID: PMCPMC3894962.

57. Phillips BE, Garciafigueroa Y, Engman C, et al. Tolerogenic dendritic cells and T-regulatory cells at the clinical trials crossroad for the treatment of autoimmune disease; emphasis on Type 1 diabetes therapy. Front Immunol. 2019;10:148. PubMed PMID: 30787930; PubMed Central PMCID: PMCPMC6372505.

58. Dalod M, Chelbi R, Malissen B, et al. Dendritic cell maturation: functional specialization through signaling specificity and transcriptional programming. Embo J. 2014 May 16;33(10):1104-1116. PubMed PMID: 24737868; PubMed Central PMCID: PMCPMC4193918.

59. D'Amour KA, Agulnick AD, Eliazer S, et al. Efficient differentiation of human embryonic stem cells to definitive endoderm. Nat Biotechnol. 2005 Dec;23(12):1534-1541. PubMed PMID: 16258519.

- This pioneering paper reports the first succesful attempt to invitro generate $\beta$-cell precursors from embryonic stem cells.

60. Kroon E, Martinson LA, Kadoya K, et al. Pancreatic endoderm derived from human embryonic stem cells generates glucose-responsive insulin-secreting cells in vivo. Nat Biotechnol. 2008;26:443-452.

61. D'Amour KA, Bang AG, Eliazer S, et al. Production of pancreatic hormone-expressing endocrine cells from human embryonic stem cells. Nat Biotechnol. 2006;24:1392-1401.

62. Rezania A, Bruin JE, Riedel MJ, et al. Maturation of human embryonic stem cell-derived pancreatic progenitors into functional islets capable of treating pre-existing diabetes in mice. Diabetes. 2012 Aug;61(8):2016-2029. PubMed PMID: 22740171; PubMed Central PMCID: PMCPMC3402300

63. Rezania A, Bruin JE, Arora $P$, et al. Reversal of diabetes with insulin-producing cells derived in vitro from human pluripotent stem cells. Nat Biotechnol. 2014;32:1121-1133.

64. Pagliuca FW, Millman JR, Gurtler M, et al. Generation of functional human pancreatic beta cells in vitro. Cell. 2014 Oct 9;159 (2):428-439. PubMed PMID: 25303535; PubMed Central PMCID: PMCPMC4617632.

65. Russ HA, Parent AV, Ringler JJ, et al. Controlled induction of human pancreatic progenitors produces functional beta-like cells in vitro. Embo J. 2015;34:1759-1772.

66. Rosado-Olivieri EA, Anderson K, Kenty $\mathrm{JH}$, et al. YAP inhibition enhances the differentiation of functional stem cell-derived insulinproducing $\beta$ cells. Nat Commun. 2019;10:1464.

67. Velazco-Cruz L, Song J, Maxwell KG, et al. Acquisition of dynamic function in human stem cell-derived $\beta$ cells. Stem Cell Reports. 2019;12:351-365. 
68. Sharon N, Vanderhooft J, Straubhaar J, et al. Wnt signaling separates the progenitor and endocrine compartments during pancreas development. Cell Rep. 2019;27:2281-2291.e5.

1050 69. Veres A, Faust AL, Bushnell HL, et al. Charting cellular identity during human in vitro $\beta$-cell differentiation. Nature. 2019;569:368-373.

70. Shahjalal HM, Abdal Dayem A, Lim KM, et al. Generation of pancreatic $\beta$ cells for treatment of diabetes: advances and challenges. Stem Cell Res Ther. 2018:9:355. PubMed PMID: 30594258.

- Review which aptly describes the current situation of using iPSC-dervied $\beta$-cells in various perspectives along with their shortcomings.

71. Henry RR, Pettus J, Wilensky J, et al. Initial clinical evaluation of VC01TM combination product-a stem cell-derived islet replacement for Type 1 Diabetes (T1D). Diabetes. 2018;67:138-OR.

72. Chayosumrit M, Tuch B, Sidhu K. Alginate microcapsule for propagation and directed differentiation of hESCs to definitive endoderm. Biomaterials. 2010 Jan;31(3):505-514. PubMed PMID: 19833385.

1065 73. Tuch BE, Keogh GW, Williams LJ, et al. Safety and viability of microencapsulated human islets transplanted into diabetic humans. Diabetes Care. 2009 Oct;32(10):1887-1889. PubMed PMID: 19549731; PubMed Central PMCID: PMCPMC2752920.

74. Calafiore R, Basta G, Luca G, et al. Microencapsulated pancreatic islet allografts into nonimmunosuppressed patients with type 1 diabetes: first two cases. Diabetes Care. 2006 Jan;29(1):137-138. PubMed PMID: 16373911.

75. Calafiore R. Microencapsulation for cell therapy of type 1 diabetes mellitus: the interplay between common beliefs, prejudices and real progress. J Diabetes Investig. 2018 Mar;9(2):231-233. PubMed PMID: 29215800; PubMed Central PMCID: PMCPMC5835460.

76. Takahashi K, Yamanaka S. Induction of pluripotent stem cells from mouse embryonic and adult fibroblast cultures by defined factors. Cell. 2006;126:663-676. PubMed PMID: 16904174.

1080 77. Teo AKK, Wagers AJ, Kulkarni RN. New opportunities: harnessing induced pluripotency for discovery in diabetes and metabolism. Cell Metab. 2013;18:775-791. PubMed PMID: 24035588.

78. Tateishi K, He J, Taranova O, et al. Generation of insulin-secreting islet-like clusters from human skin fibroblasts. J Biol Chem. 2008;283:31601-31607. PubMed PMID: 18782754.

79. Bai $Q$, Desprat $R$, Klein $B$, et al. Embryonic stem cells or induced pluripotent stem cells? A DNA integrity perspective. Curr Gene Ther. 2013;13:93-98. PubMed PMID: 23317057.

80. Kondo $\mathrm{Y}$, Toyoda T, Inagaki N, et al. iPSC technology-based regenerative therapy for diabetes. J Diabetes Investig. 2018;9:234-243. PubMed PMID: 28609558.

81. Pagliuca FW, Melton DA. How to make a functional -cell.. Development. 2013;140:2472-2483. PubMed PMID: 23715541.

82. Alagpulinsa DA, Cao JJL, Driscoll RK, et al. Alginatemicroencapsulation of human stem cell-derived beta cells with CXCL12 prolongs their survival and function in immunocompetent mice without systemic immunosuppression. Am J Transplant. 2019 Jul;19(7):1930-1940. PubMed PMID: 30748094. .
- In this report, authors elucidate a novel approach in transplanting human stem cell-derived $\beta$-cells that can improve tranplantation efficiency and safety.

83. Omole $A E$, Fakoya AOJ. Ten years of progress and promise of induced pluripotent stem cells: historical origins, characteristics, mechanisms, limitations, and potential applications. PeerJ. 2018;6:e4370.

84. Medvedev SP, Shevchenko Al, Zakian SM. Induced pluripotent stem cells: problems and advantages when applying them in regenerative medicine. Acta naturae. 2010;2:18-28. PubMed PMID: 22649638.

85. Warlich E, Kuehle J, Cantz T, et al. Lentiviral vector design and imaging approaches to visualize the early stages of cellular reprogramming. Mol Ther. 2011;19:782-789. PubMed PMID: 21285961.

86. González F, Boué S, Belmonte JCl. Methods for making induced pluripotent stem cells: reprogramming à la carte. Nat Rev Genet. 2011;12:231-242. PubMed PMID: 21339765.

87. Doi D, Samata B, Katsukawa $M$, et al. Isolation of human induced pluripotent stem cell-derived dopaminergic progenitors by cell sorting for successful transplantation. Stem Cell Reports. 2014;2:337-350. PubMed PMID: 24672756.

88. Okubo T, Iwanami A, Kohyama J, et al. Pretreatment with a $\gamma$ secretase inhibitor prevents tumor-like overgrowth in human iPSCderived transplants for spinal cord injury. Stem Cell Reports. 2016;7:649-663. PubMed PMID: 27666789.

89. Itakura G, Kawabata S, Ando M, et al. Stem Cell Reports Ar ticle FailSafe System against Potential Tumorigenicity after Transplantation of iPSC Derivatives. 2017. doi: 10.1016/j.stemcr.2017.02.003.

90. Ben Jehuda R, Shemer $Y$, Binah O. Genome editing in induced pluripotent stem cells using CRISPR/Cas9. Stem Cell Rev Rep. 2018;14:323-336.

91. Kim K, Doi A, Wen B, et al. Epigenetic memory in induced pluripotent stem cells. Nature. 2010;467:285-290. PubMed PMID: 20644535.

92. Maehr R. iPS cells in Type 1 diabetes research and treatment. Clin Pharmacol Ther. 2011;89:750-753.

93. Sneddon JB, Tang Q, Stock $P$, et al. Cell stem cell perspective stem cell therapies for treating diabetes: progress and remaining challenges. Stem Cell. 2018;22:810-823.

94. Odorico J, Markmann J, Melton D, et al. Report of the key opinion leaders meeting on stem cell-derived beta cells. Transplantation. 2018 Aug;102(8):1223-1229. PubMed PMID: 29781950.

95. Han X, Wang M, Duan S, et al. Generation of hypoimmunogenic human pluripotent stem cells. Proc Nat Acad Sci. 2019:116:10441-10446. PubMed PMID: 31040209.

- An interesting research attempting to provide an "on-the-shelf" $\beta$-cell-like product with virtually no need of immunosuppression therapy.

96. Qadir MMF, Álvarez-Cubela S, Belle K, et al. A double fail-safe approach to prevent tumorigenesis and select pancreatic $\beta$ cells from human embryonic stem cells. Stem Cell Reports. 2019;12:611-623. .

- Qadir and colleagues designed an elegant strategy to generate $\beta$ like cells equipped with a double fail-safe mechanism preventing tumorigenesis and increasing differentiation efficiency.
1100

1105 